\title{
Research on the Strategic Management of the Leading Enterprises of Sichuan Liquor under the New Economic Background
}

\author{
Dongchuan Lin \\ Sichuan Agricultural University \\ Chengdu, China \\ Xueke Chen \\ Sichuan Agricultural University \\ Chengdu, China
}

\author{
Tianyu Xie \\ Sichuan Agricultural University \\ Chengdu, China \\ Shanxiang Liang \\ Sichuan Agricultural University \\ Chengdu, China
}

\begin{abstract}
With the liquor industry stepping into the "new normal" period, and being affected by the factors, like " three public consumption limitation", "eight provisions" as well as industry overcapacity, more risks start to occur the development. This paper analyzes the current situation of liquor industry in Sichuan Province comprehensively, then analyzes the environment of liquor industry based on PEST method, and proposes the hierarchical strategy in liquor industry on strategic transformation of leading enterprises in Sichuan province with the new requirements such as "Internet Plus" and supply side reform.
\end{abstract}

Keywords—strategic management; Internet plus; new normal; supply side reform

\section{INTRODUCTION}

In recent years, China's economic development has shifted from high-speed growth to medium-high speed of the "new normal" period. After liquor industry that is regarded as the consumer goods industry passing through the initial adjustment, the Chinese liquor industry started to set about a more in-depth adjustment since 2014 under the influence of superposition, such as the macroeconomic policy, overcapacity, economic downturn and etc. The industry officially entered the new era with the coexistence of the opportunity and Challenge. Under the market economy environment nowadays, liquor enterprises are facing more intense market competition. With the occurrence of liquor plasticizer incident and the gradual introduction of "ban wine" announced by the central and the local government, combined with the Central Military Commission from the tight three public consumption of governance, Wuliangye Group and other high-end liquor sales have been seriously hampered. In the face of difficulties, the dilemma of how to adapt to the new normal environment and the

This paper is supported by the general project of Sichuan Center for Liquors Development Research in 2015 (NO.CJY15-06), and by the funding of the Discipline Construction and the Academic Team Construction program of SICAU. implementation of strategic transformation has realistic research significance.

\section{OVERVIEW OF SICHUAN LIQUOR INDUSTRY DEVELOPMENT}

Sichuan liquor industry has made great contributions to the economic and social development of Sichuan Province with unique geographical advantages and brewing technology from generation to generation, especially in stimulating investment and consumption, promoting local economic development, solving labor employment and increasing farmers' income as well as new urbanization etc.. Wuliangye, Luzhou Tequ Jiannanchun, Langjiu, Quanxing Daqu, Tuopai Qujiu was named as the national wines in Sichuan Province. In 2015, the liquor business which was above the scale of Sichuan province achieved the main business sales revenue 105.681 billion RMB, with year-onyear growth of $33.09 \%$. But under the internal problems, like the effects of domestic and international economic decreasing trends, the supply and demand contradictions in liquor industry, structural imbalance, and product quality appearing varying greatly and traditional marketing, Sichuan liquor industry is facing severe challenges. Overcapacity is the primary risk in the field of liquor production, coupled with the weak trend of sales in the whole industry. At the same time, the industry is highly competitive with the rising cost of circulation, and the circulation of liquor has an imbalance. At the same time, liquor product structure is unreasonable. Enterprises blindly pursue high-end products, chasing high-priced wine production, consequently be away from the public demand. As for the changing of modern consumer taste, many liquor companies do not make the appropriate adjustments, and it is why adapting to new consumer trends becomes difficult. In addition, a part of the enterprises' development concept lags behind the times change under the "low threshold, high yield, less innovation" model. The domestic liquor industry has been exposed in worrying liquor quality and safety problems, such as the 
"plasticizer" event, "additives" and other events. Finally, the benefits of liquor surely decreased to a certain extent. Due to top-grade liquor sales blocked, liquor concentrated areas and wine sales revenue fell sharply, resulting in the province's liquor industry growth rate falling significantly, high-end liquor slow-moving and profit declining.Profits and taxes in liquor industry of Sichuan province appeared a negative growth for the first time in 10 years.

\section{THE LIQUOR INDUSTRY DEVELOPMENT ENVIRONMENT PEST ANALYSIS}

\section{A. Political and Legal Environment $(P)$}

Since March 26, 2012, the State Council held the fifth session of the Independent Commission against Corruption, "limit three public consumption" "ten provisions" and other policies have been introduced in succession. With the implementation of these policies as well as the gradual reduction in government and business consumption, it caused a great impact on long-term dependence on government consumption of liquor enterprises, especially top-grade liquor business. Many top-gradeliquor production enterprises sales fell significantly. Liquor enterprises that were heavily dependent on top-grade government consumption patterns began to go into the bottleneck period after country announcing to limit the development of "three public consumption ".

\section{B. Economic Environment $(E)$}

China's economic development comes into the highspeed growth from about $10 \%$ transferring to the mediumhigh speed of $7 \%-8 \%$ in new normal period. After the reform and opening up, the liquor industry ushered in the golden development period. The industry competition pattern and development trend presented new features since 2013.The liquor production and sales from high growth began to step into the medium-speed growth and the industry growth rate from $20 \% \sim 30 \%$ reduced to less than $10 \%$ on average per year. In 2014, the liquor industry showed that the increase in sales growth is lower than the increase in production for the first time in the past decade. The explosive growth of the industry, which was relying on top-grade liquor prices to stimulate a whole industry, has disappeared. Coupled with raising the costs of production and operating, industry profits generally demonstrated a decline trend.

\section{Social Environment $(S)$}

With the improvement of living standards as well as safety incidents caused by "plasticizer" "fake wine" make consumers pay more attention to "healthy consumption" and "green consumption." In addition, changes in demographic structure lead to changes in the structure of social needs. As the main consumer groups like 1980s and 1990s, they are more pursuit of personalized products, diversification, alcohol alternatives, like red wine, cocktails etc.. It would bring challenges to the development of China's traditional wine liquor industry. In coexistence of the multi-species in the market for consumers, liquor industry consumer groups gradually are shrinking.

\section{Technical Environment (T)}

The traditional liquor industry is highly dependent on the dealer, but emerging channels, like "Internet +" shows rapid development and cause a major impact on liquor traditional sales channels. It will subvert the traditional wine business rules, industrial value chain, business model and competitive landscape. Breaking the inherent rules of the past market and management model could affect the profitability of enterprises. As for other industries in China, most of them have begun to make full use of the Internet for online marketing, such as Suning Group Appliance using the "Internet +" power platform for a successful transformation to provide valuable experience of China's liquor industry. Sichuan liquor enterprises should change their sales model according to changes in consumption patterns as well. Injecting new marketing ideas such as the big data marketing, Internet thinking in order to expand sales channels and promote the diversification of industry channels to improve market share.

\section{THE STRATEGY OF TRANSFORMATION OF LEADING ENTERPRISES IN SICHUAN WINE}

\section{A. The Overall Strategic Transformation}

1) Adoption of diversified business strategy: For many companies, especially those who have achieved good results in an industry tend to take a diversified strategic transformation, involving industry-related or even completely different industries. Many world's leading companies are in the implementation of diversification strategy, such as automotive companies, like GM, Ford or household appliances companies, like Sony, Panasonic. As a leading position in the liquor industry including Wuliangye Group, Luzhou Laojiao Group also want to get involved in more areas in order to achieve expansion, so diversification is the kind of business to achieve the expansion of a strategy.

2) Adoption of market development strategy: "Limit three public consumption" "Ten provisions" and other policies have been introduced, while government consumption and business consumption gradually reduced. Therefore, Sichuan wine enterprises should turn to adhere to the principle of market-oriented, and constantly adjust the strategic positioning to adapt to the current economic development. Entreprises should look for new target markets, such as low-end market, so as to stimulate sales and to make up for shrinking losses of government market.

\section{B. Business Layer Strategy Transformation Strategy}

1) Implementing the differentiation strategy: There is current wine business thinking on how to cater to different consumer groups, the production of more intimate and cordial products to win the public goodwill. Sichuan wine enterprises should launch personalized custom wine to meet the different consumer groups and the needs of different consumer groups as well as making their own products more unique. 
2) Implementing cost leadership strategy: Cost leadership requires enterprises to establish efficient production facilities on the basis of experience to go all out to reduce costs so as to pay attention to the control of the cost and management. As a result, the cost is lower than the competitors. There is always a enterprise to make the appropriate attempt among Sichuan liquor industry, in which Jiannanchun is typical. The main way to get the cost lead is to build large-scale production on a good brand image.

\section{Functional Layer Transformation Strategy}

1) Paying attention to the control of quality of production: After the alcoholic "plasticizer" incident and Fenjiu emergency recall incident, the national food safety law regulations will be more severe. 13 enterprises of Sichuan in total were named and shamed by the State Food and Drug Administration. At the same time, with the improvement of consumer health awareness, the quality of liquor will be controlled stringently. Wuliangye increased the expenditure of product development as well as establishing monitoring institution within the enterprise. Through the product technology, enterprise supervision, supervision and inspection of the investigation and acceptance of three dynamic differences in supervision means, Yibin Wuliangye quality has been a higher guarantee.

2) Broadening the sales channels, injecting into the ebusiness platform: Before government clearly limits the "three public consumption", the liquor industry has been in a state with excess capacity and sales channel deformity. Enterprises should establish a one-stop service with consumers as well as ensuring quality to win the trust of the market and consumers from the sales channels and continuous innovation. From the original simple product sales which is relying on the governmental purchase channel to transfer into experiential marketing, and pay attention to the needs of consumers in all directions .It can meet the international high-end wine sales model.

3) Organizational Structure Transformation: Transition from a controlled organization to a market organization. The deliberate model of organization is the organizational model of organizational structure, function and management in which the value of the strong side is dominant. There are organizational maladies on multi-level, strong command, separation (vendor cooperation) and heavy power. Now the development of enterprises encountered bottlenecks, so the structure of this organization, management, performance and other aspects have been unable to meet the needs of the current market competition. Only the market-oriented organizational model is often the most competitive, anti-risk, dynamical, sustainable and do not fluctuate with environmental changes.

\section{REFERENCES}

[1] Chen Yuhou. A Study on the Regional Cooperation of "Golden Triangle of Chinese Liquor"[J].Journal of Sichuan University of Science and Technology (Social Science Edition),2012,(01):57-60.

[2] Jiang Jia. Strategies for Deep Adjustment of Mingyou Liquor Enterprises-take Sichuan as an example[J].Journal of Sichuan University of Science and Technology (Social Science Edition),2014,(03):123-146.

[3] Yang Xianhua, Yang Guanrong, Huang Zhiyu, Zhou Ling, Huang Hai, Zhao Fukui. A Review of the Relationship between Chinese Liquor and Human Health[J].Wine,2014,05:10-13.

[4] $\mathrm{Xu}$ Jing. Double liquor brand competition in the new situation[J].Public Relations World,2014,01:82-83.

[5] Zhang Juan,Tan Yongli.Current Situation and Development Trend of Liquor Industry in China[J].Time Economic and Trade, 2007,(07):7576. 